\title{
Electromagnetic Compatibility Analysis of High-Power Pulse Drive Source
}

\author{
Wuxi Leng1, Junxu He ${ }^{2}$ \\ ${ }^{1}$ Electronic Warfare Academy, National University of Defense Technology, Hefei, China \\ ${ }^{2}$ School of Electrical Engineering, Zhejiang University, Hangzhou, China \\ Email: xiguaabigpotato@163.com
}

How to cite this paper: Leng, W.X. and He, J.X. (2020) Electromagnetic Compatibility Analysis of High-Power Pulse Drive Source. Journal of Power and Energy Engineering, 8, 32-44.

https://doi.org/10.4236/jpee.2020.87003

Received: June 24, 2020

Accepted: July 28, 2020

Published: July 31,2020

Copyright $\odot 2020$ by author(s) and Scientific Research Publishing Inc. This work is licensed under the Creative Commons Attribution International License (CC BY 4.0).

http://creativecommons.org/licenses/by/4.0/ (c) (i) Open Access

\begin{abstract}
Due to the widespread application in recent years, high-power pulse sources have received special attention from large companies and R \& D institutions around the world. Compared with foreign mid-range and high-end products, China's development in this field is relatively lagging behind and lacks mature mid-range and high-end products. The reason is not only because of lack of theoretical support, but more importantly, in the pursuit of indicators and design, the electromagnetic compatibility of the equipment has not been studied thoroughly, resulting in a large degree of distortion in the output waveform, resulting in a reduction in actual value. This paper introduces the sources of high-power pulsed electromagnetic interference of the main driving source, conducts a reasonable high-power electromagnetic compatibility driving source dynamic analysis, and proposes measures to improve electromagnetic compatibility.
\end{abstract}

\section{Keywords}

Pulse Drive Source, Electromagnetic Compatibility, High Frequency Pulse

\section{Introduction}

Electromagnetic compatibility, as a method of solving electromagnetic interference, has attracted more and more attention. Electromagnetic compatibility refers to the ability of a device or system to work normally in a specific electromagnetic environment without causing irresistible electromagnetic interference to the external device environment. If electronic equipment is to operate stably in an electromagnetic environment, it is necessary to solve the electromagnetic interference caused by the external environment and the equipment itself. Three conditions are required to produce electromagnetic interference: one is the 
source of electromagnetic interference, the other is the method of electromagnetic interference, and the third is equipment sensitive to electromagnetic interference. In recent years, the application range of ultrashort wave pulses has become wider and wider. In the field of lidar and optical fiber communication, such a driving source capable of generating large, narrow, and pulse currents is necessary. Its excellent performance and broad application prospects have attracted the attention of large companies and scientific research institutions. The high-power pulse driving source as the pre-driving of ultrashort wave laser has also become a research hotspot.

This paper studies a high-power pulse control source. For this pulse drive source based on a high-frequency circuit, the quality of electromagnetic compatibility directly determines the quality and stability of the output pulse of the device. In the control process of the pulsed semiconductor laser, the waveform quality and stability of the laser output laser are directly determined by the control pulse. The optimization of the electromagnetic compatibility of the pulse drive source can indirectly improve the quality of the driven laser output. When applied to the field of energy exploration, the most ideal pulse wave form can improve the accuracy of controlled exploration instruments. It can be said that solving the electromagnetic interference problem of the high-power pulse drive source is an essential prerequisite for improving product performance. In summary, it is of practical significance to study the electromagnetic compatibility of high-power pulse drive sources. While seeking higher output amplitude and narrower pulse width, the quality of its pulse output waveform cannot be ignored. This usually becomes a key factor in determining whether the drive device is working properly.

\section{Pulse Power Source and Electromagnetic Compatibility Optimization}

\subsection{Introduction of Common Pulse Power Sources}

According to the different energy storage methods, pulse power systems can be divided into capacitive energy storage types and inductive energy storage types [1]. The advantage of the inductive energy storage pulse power device is that the energy storage density is high, it is easy to miniaturize, and it can be used in conjunction with the magnetic explosion compression device. However, its disadvantages are: 1) a large-capacity circuit breaker is required during the conversion of the load energy, and these switches must work quickly and reliably; 2) the energy conversion efficiency of the single-layer inductive energy storage device is low, although many The use of layered inductance energy storage may increase efficiency, but it complicates circuits or equipment, thereby increasing congestion and cost. The capacitor energy storage pulse power supply has the advantages of high energy conversion efficiency, adjustable pulse width, strong repetitive frequency working ability, wide impedance adjustment range, and good adaptability to microwave sources [2]. Due to the attractive energy storage 
capacitors of pulse generators, in recent years, on the basis of higher power guarantees, the miniaturization of research and the operating frequency of capacitive energy storage devices have attracted wide attention from researchers., Nationwide and overseas.. Capacitor energy storage pulse generators are mainly divided into four categories: primary energy storage devices, booster devices, pulse forming devices and loads. According to different boosting devices, capacitor energy storage pulse generators can be divided into three types: Marx type, transformer type and transmission line voltage multiplier type. The following mainly introduces the types of Marx capacitive transformers and energy storage pulse generators.

\subsubsection{Transformer Pulse Generator}

The most widely used pulse drive source is a transformer-type pulse generator. The basic principle is that the primary energy storage capacitor discharges the primary winding of the transformer, thereby generating resonance in the primary circuit [3]. Due to the tight coupling between the primary winding and the secondary winding, a high-voltage pulse signal is sent out in the secondary winding to charge the pulse forming line. When the load of the secondary winding reaches a certain voltage value, the main switch is activated, the secondary winding releases the load, and a high-voltage pulse exceeding several hundred kilovolts is generated on the load. The pulse generator has small volume and high repetitiuality are highon frequency, which can reach more than $100 \mathrm{hz}$. According to the structure of the iron core, the pulse transformer can be divided into a closed-loop magnetic core pulse transformer, an air-core resonant transformer and an open-loop magnetic core Tesla transformer. Closed-loop magnetic core transformers have a high coupling coefficient and transmission efficiency, but the volume and qunder high voltage and repetition frequency, and the operation is difficult. 1.2 MV high voltage pulse transformer (Figure 1) (2009) was developed by Ma Chenggang and others. The Chinese Academy of Physical Engineering uses 14 large iron rings made of amorphous iron-based materials as magnetic cores. The size of a single magnetic ring is $780 \times 620 \times 25 \mathrm{~mm}$, the speed ratio is 2:54, the primary charge is $56 \mathrm{kV}$, and the pulse generator it drives is larger than $6 \mathrm{~m}^{3}$ and weighs more than 4 tons.

The air-core transformer is light in weight, small in size, has no iron loss, and no magnetic saturation, but the coupling coefficient is low. Figure 2 is a $500 \mathrm{kv}$ cylindrical hollow pulse transformer. It was $248 \mathrm{~mm}$ in diameter and $700 \mathrm{~mm}$ in length in South Korea in 2005. The main input voltage is $50 \mathrm{kv}$, and the secondary input voltage can produce a high-voltage pulse voltage of $500 \mathrm{kv}$, with a conversion ratio of 1:10. Tesla transformer with open core structure has a high coupling coefficient (up to 0.9 or above) and good insulation conditions. Under repeated operating conditions, it is almost immune to nuclear saturation and frequency limitations. The Russian Institute of High Current Electronics first used an open-loop magnetic core to closely combine the transformer and the pulse forming line (PFL) to achieve the integration of high-voltage pulse genera- 
tion and formation. Figure 3 shows the "SINUS-120" pulse generator developed by Gubanov et al. The integration of Tesla transformer and coaxial line makes the generator structure more compact. It can produce a suitable burden on 200 $\mathrm{kV} \Omega 30$ and a nanosecond pulse high voltage pulse width of 4 repetition rate up to $1000 \mathrm{pps}$; the structure of "RADAN-303B" is shown in Figure 4. The Tesla transformer is built between the center tube and the outer tube of the Bloomline. It can produce $150 \mathrm{kv}$ under the proper load, and the pulse width is $4 \mathrm{~ns}$. The voltage pulse can work under high frequency and weighs only $28 \mathrm{~kg}$.

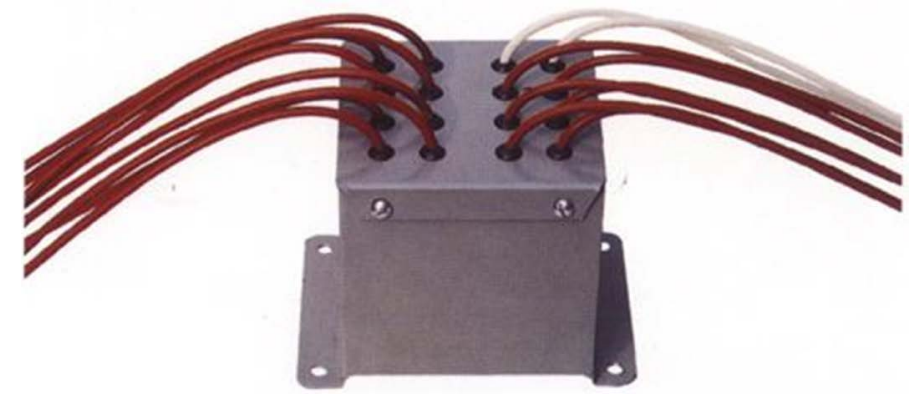

Figure 1. 1.2 MV high voltage pulse transformer.

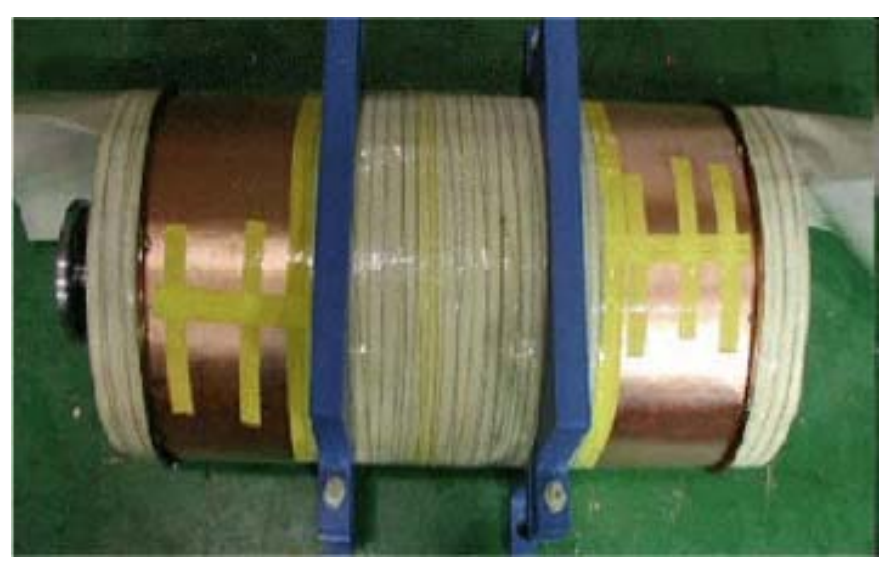

Figure 2. Air core pulse transformer.

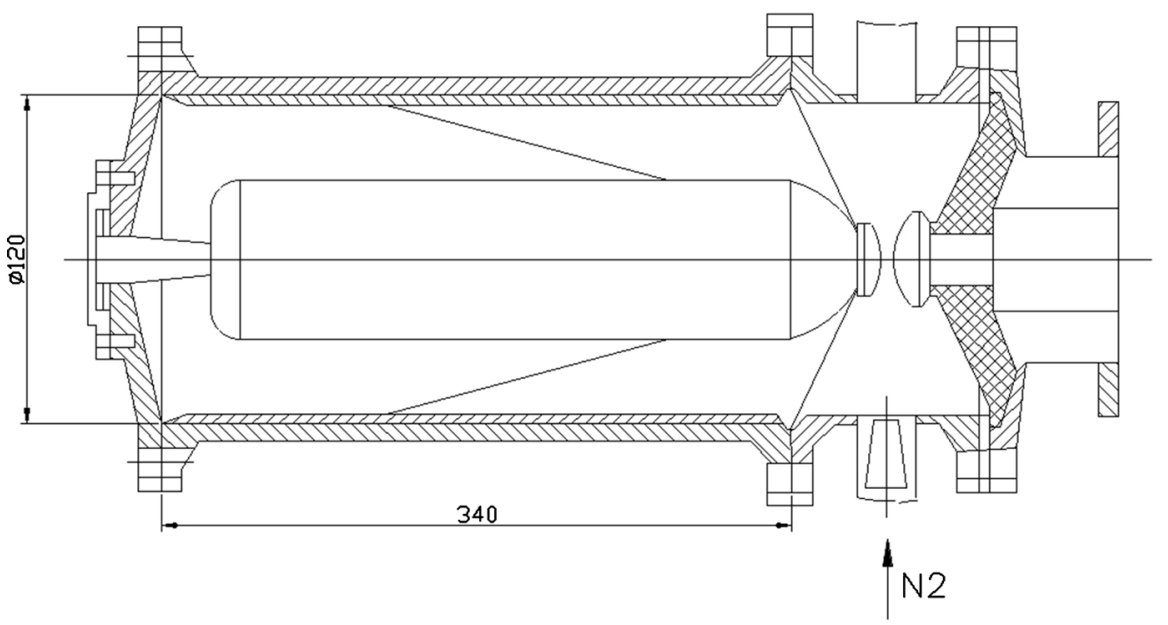

Figure 3. "SINUS-120" structure diagram. 


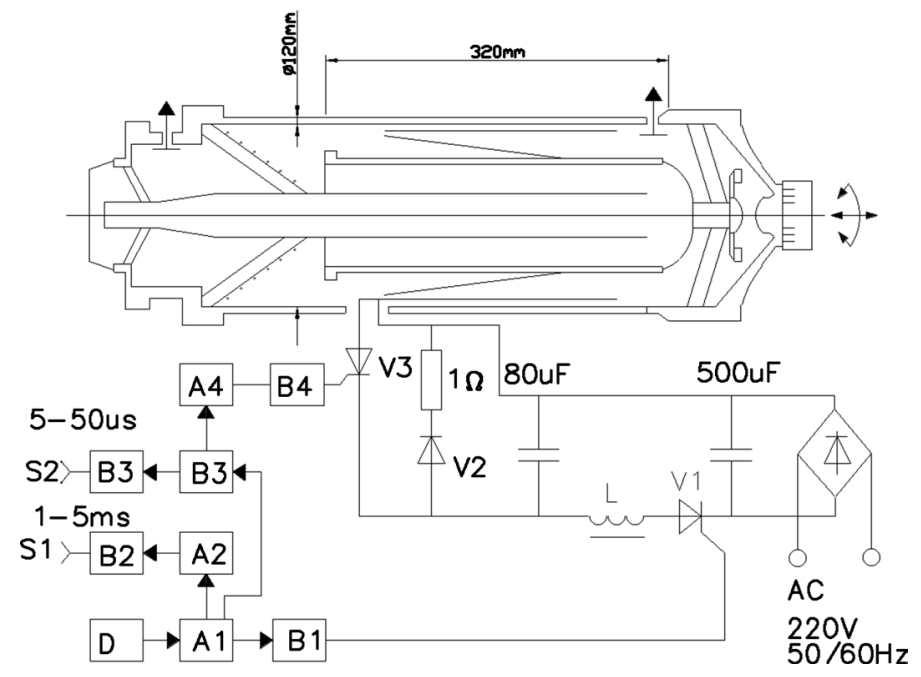

Figure 4. "RADAN-303B" structure diagram.

\subsubsection{Marx Type Pulse Generator}

The basic principle of the Marx pulse generator can be briefly summarized as follows. Several capacitors are charged in parallel and then discharged in series to generate high-voltage pulses. The related concept was proposed by Professor MarxE in 1923, and was later named "Marx generator", and obtained the patent right, which was the first time it was used in the lightning tension experiment. In the 1960s, J.C. Martin successfully combined the pulse width of tens of nanoseconds combined with the Marx generator online. The latter has been used to generate "Marx particle beam type high-power pulsed X-rays. The high-power pulse system is developing rapidly.

\subsection{Technical Key to Optimization of Electromagnetic Compatibility of High-Power Pulse Drive Source}

The central circuit of the high-power pulse control source is a high-frequency circuit, and the narrow-band pulses of high-power output contain abundant harmonics. Optimizing the electromagnetic compatibility of this circuit is a challenge for researchers. Electromagnetic interference can be divided into conducted interference and radiated interference. For conducted interference, the rational design of high-frequency printed circuit boards (PCBs) has become an important part. The use of filters and adaptive circuits, the choice of component types, PCB boards and good grounding design cannot be ignored. Shielding measurement based on circuit characteristics is an effective method to eliminate radiated interference. For high frequency circuits, many parameters that can be ignored in the low frequency band must be considered. How to analyze high-frequency circuit parameters to optimize the circuit has also become a problem. While optimizing electromagnetic compatibility, the optimization of the circuit output pulse index is also complementary. Finally, the improved high-power pulse source test method is also a key link. Many designers actually design relatively ideal products, but the results are not ideal due to incorrect 
measurement methods. In summary, the optimization of electromagnetic compatibility is not just a theoretical issue: only by analyzing and experimenting on products at a certain theoretical level can we obtain high-power pulse sources with high-quality output.

\subsection{Electromagnetic Compatibility Test of High-Power Pulse Drive Source}

Because the time and frequency domain characteristics of electromagnetic interference are very complex, the current mathematical modeling and computer simulation techniques have certain limitations. In some cases, electromagnetic compatibility must be tested through specific experiments, and electromagnetic compatibility tests were conducted. The electromagnetic compatibility test is generally divided into the following aspects: the second is the anti-static discharge capacity test; the third is the anti-overvoltage test; the fourth is the pipeline disturbance caused by the radio frequency immunity test field. The purpose of the fast transient electrical pulse group immunity test is to simulate the disturbance of transient pulses generated during the operation of switches, relays and other equipment. According to the evaluation of the test level, a specific test voltage is attached to different types of lines or ports, and the evaluation is performed according to the test results. The electrostatic discharge immunity test is to simulate the discharge phenomenon when workers touch the equipment. A capacitor of $150 \mathrm{pF}$ can usually be used to simulate the ability of the human body. A discharge resistance of $330 \Omega$ is used to simulate the resistance of the human body, and contact discharge or air discharge can be based on the description of the device. The purpose of the anti-overvoltage test is to simulate the interference of lightning strikes to the equipment. The immunity test of radio frequency field induced disturbance-the injection current test is designed to simulate the influence of electromagnetic field induced current on various sensitive parts of the device. In the process of optimizing the design of the high-power pulse drive source, reasonable grounding technology is used to effectively prevent the interference of electrostatic discharge on the device. Through continuous optimization, the high-power pulse source drive device has certain electromagnetic immunity and can work in a typical industrial environment. However, in the face of a difficult industrial environment, the equipment is at risk of failure.

\section{Electromagnetic Interference and Approaches of High-Power Pulse Drive Source}

\subsection{Sources of Electromagnetic Interference}

\subsubsection{High-Order Harmonic Interference on the Input Side of the Pulse Drive Source}

The input part of the pulse control source is a rectifier circuit with nonlinear characteristics. Therefore, higher harmonics are generated, which distort the input voltage and current waveforms [4]. Figure 5 shows the input voltage and 
current waveforms. Taking phase a as an example, it can be seen that the input current waveform has been deformed.

After a spectral analysis of the input current, it can be seen that the $5 \mathrm{e}$ and $7 \mathrm{e}$ harmonic components are very large and not much smaller than the fundamental component. As shown in Figure 6, the ordinate is the amplitude, and the basis of the harmonic component is the ratio of the amplitude of the wave component. These higher harmonics will interfere with the input power system [5].

\subsubsection{High-Order Harmonic Interference on the Output Side of the Pulse Drive Source}

The interference on the output side of the pulse control source is formed by pulse width modulation PWM, which is related to the level of the pulse width modulation frequency and the pulse width modulation process. For example, the pulse control sources GTR, GTO, and SCR use low frequency modulation, high harmonics, and large low-frequency parts, which are easy to be heard by the human ear; the IGBT pulse width modulation frequency is high, up to $20 \mathrm{khz}$, the modulation frequency is high, and the low-frequency harmonics are small. The human ear cannot hear. However, high-frequency signals exist objectively. The output voltage and current waveforms of the pulse control source are shown in Figure 7.

For the form of voltage and current waves, the content of each harmonic can be obtained by Fourier analysis. The higher harmonic current directly interferes

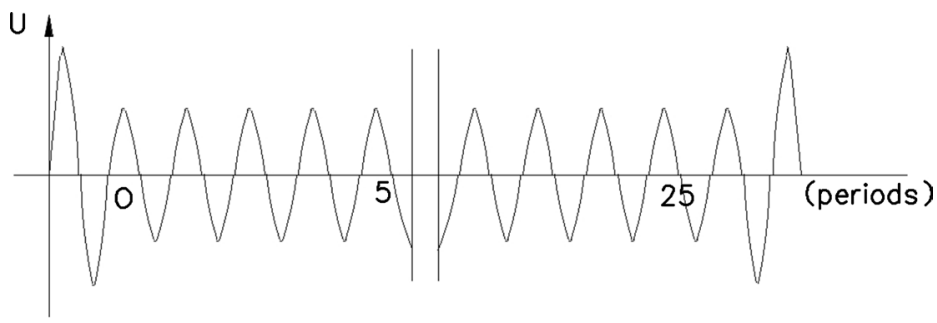

Figure 5. Waveform distortion of input current.

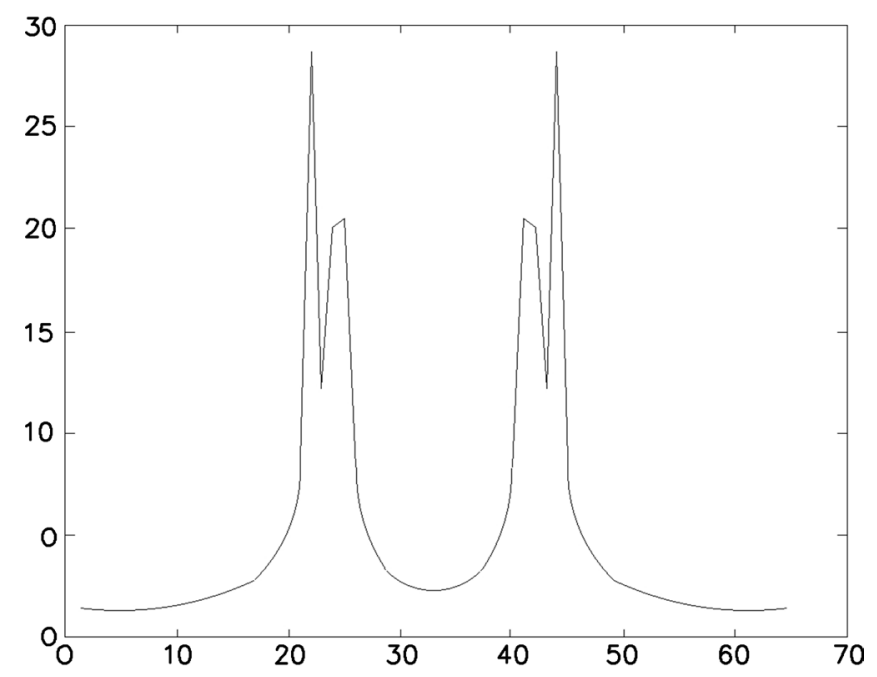

Figure 6. Spectrum analysis of input current. 
with the load, and the higher harmonic current also radiates into the space through the cable, disturbing the adjacent electrical equipment.

\subsubsection{Interference Caused by Various Conditions of the Power Grid When the Pulse Drive Source Is Put into Operation}

1) The pulse drive source is connected to the distribution network. When the three-phase voltage of the distribution network is unbalanced, the waveform of the input current of the pulse drive source is deformed. The three-phase voltage of the distribution network is unbalanced, and the unbalance ratio $=[($ single-phase maximum single-phase minimum)/three-phase average voltage] $\times$ 100. At this stage, the input voltage and current waveforms are the peak values of the input current 1 , as shown in Figure 8.

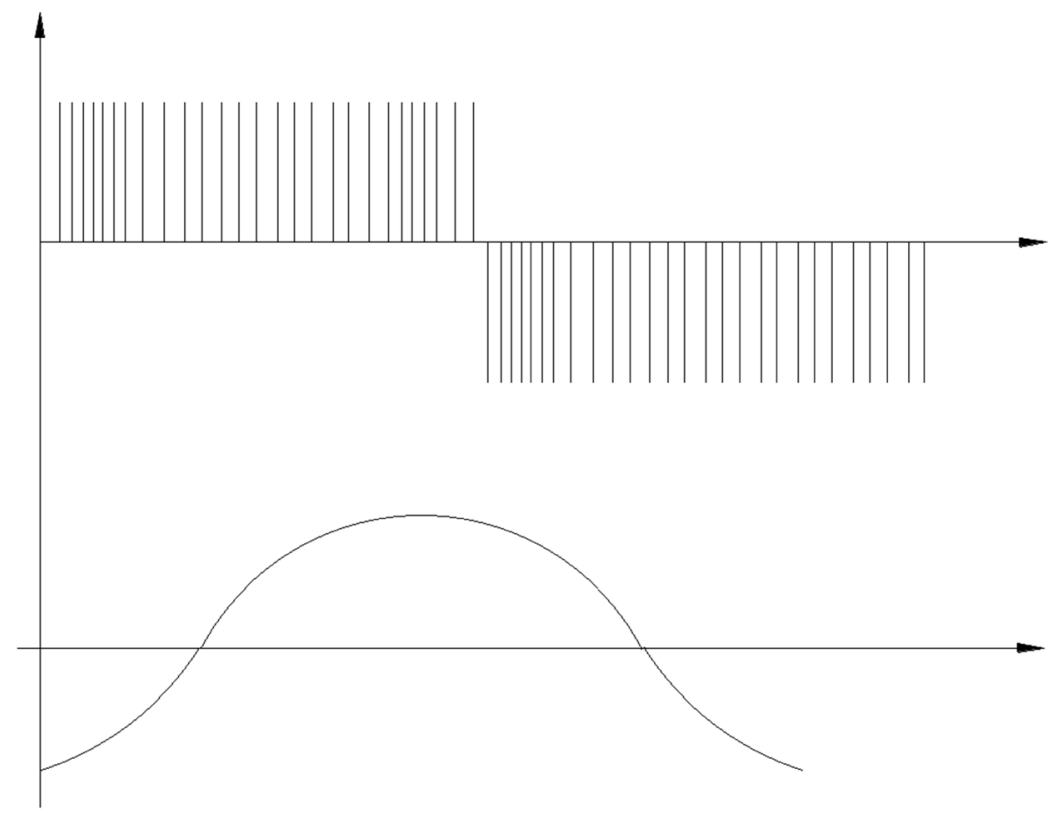

Figure 7. Pulse drive source output voltage and current waveform.
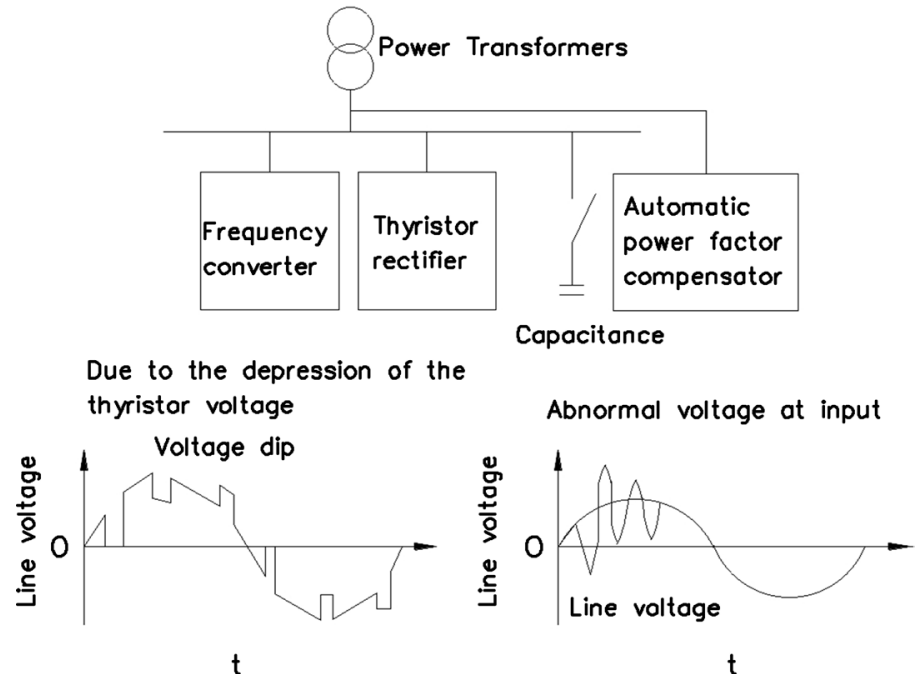

Figure 8. The distribution network is connected with capacitors and rectifiers. 
2) The pulse drive source is connected to the distribution network, and the distribution network transformer is connected to the power factor compensation capacitor, thyristor rectifier, etc. [6]. At this point, the pulse drive source is connected to the distortion of the current waveform. The pulse drive source is connected to the network at the same time, as shown in Figure 8. At this time, the waveform of the input voltage of the pulse control source is deformed, and the peak value of the input current increases, which increases the voltage load of the correction diode.

\subsubsection{Sensitive Components}

In the pulse drive source system, each electronic component and component can become a sensitive disturbance unit. However, electromagnetic interference occurs only when the signal from the interference source exceeds the allowable range of the electronic component, and the electronic component can respond to the frequency of the signal from the interference source. Obviously, in order to improve the electromagnetic compatibility of the system, we must start from these two aspects.

\subsection{Interference Signal Propagation}

Electromagnetic interference signals can be coupled from the interference source to the sensitive unit in various ways, generally divided into the following three ways:

1) Coupling of conductors: Interference signals propagate through conductors such as power lines, signal lines, and control lines;

2) Co-impedance coupling: The interference signal interferes with other circuits through the current in the common impedance. The main reason for this generation is that the grounding method is not good;

3) Radiation coupling: The radiated electromagnetic field caused by the movement of charges in components or wires can be divided into near field and far field. The far-field coupling method is mainly magnetic radiation; the near-field coupling is divided into capacitive coupling (caused by distributed capacity) and inductive coupling (caused by mutual inductance). In VFD mode, high-speed switching of the main circuit is inevitable. In VFD, radiated interference is the most serious, usually transmitted in four ways:

1) Direct conduction along the wire;

2) Capacitive coupling of the interference source wire and other nearby wires:

3) Inductive coupling between wires:

4) Due to the "antenna effect", the exposed shaft radiates directly.

\section{Electromagnetic Compatibility Analysis and Design of Pulse Drive Source}

The pulse control source needs to operate in an industrial environment with high electromagnetic interference (EMD). In this case, it is both a source of electromagnetic radiation and a receiver of electromagnetic radiation. 


\subsection{Pulse Drive Source as Noise Emission Source}

The parasitic capacity C exists inside the motor cable and the motor, and generates a high-frequency pulse noise current I through the parasitic capacity. At this point, the pulse drive source becomes a radiation source. Since the current source 1 of radiated interference is an inverter, it must return to the pulse control source. In Figure 9, Z = is the ground impedance, and $\mathrm{Z}$ is the impedance between the power supply cable and ground. The voltage drop caused by the noise current of these two impedances will affect other devices on the same network and cause interference. In addition, the correction part of the pulse control source will also generate low-frequency harmonics, resulting in distortion of the grid voltage. When the grid short-circuit impedance is less than $1 \%$, it is recommended to increase the line inductance to suppress low-frequency interference high-frequency interference current I. If an unshielded motor cable is used, then the drive source of high-frequency noise signal current pulse is on an unknown path, and the generation of high-frequency components of voltage drop is in this cycle, affecting other devices. In order to return the high-frequency noise current I to the pulse drive source along a given path, a shielded traction cable as shown in Figure 10 is required. The shielding layer of the cable should be connected to the pulse drive source housing and the motor housing. In this case, high-frequency noise can be eliminated.

\subsection{Pulse Drive Source as Noise Receiver}

In Figure 11, the high-frequency noise current enters the pulse drive source through the potential and the coupling capacitor, and a voltage drop occurs at the impedance $Z$ terminal, which may cause disturbance noise. At this point, the

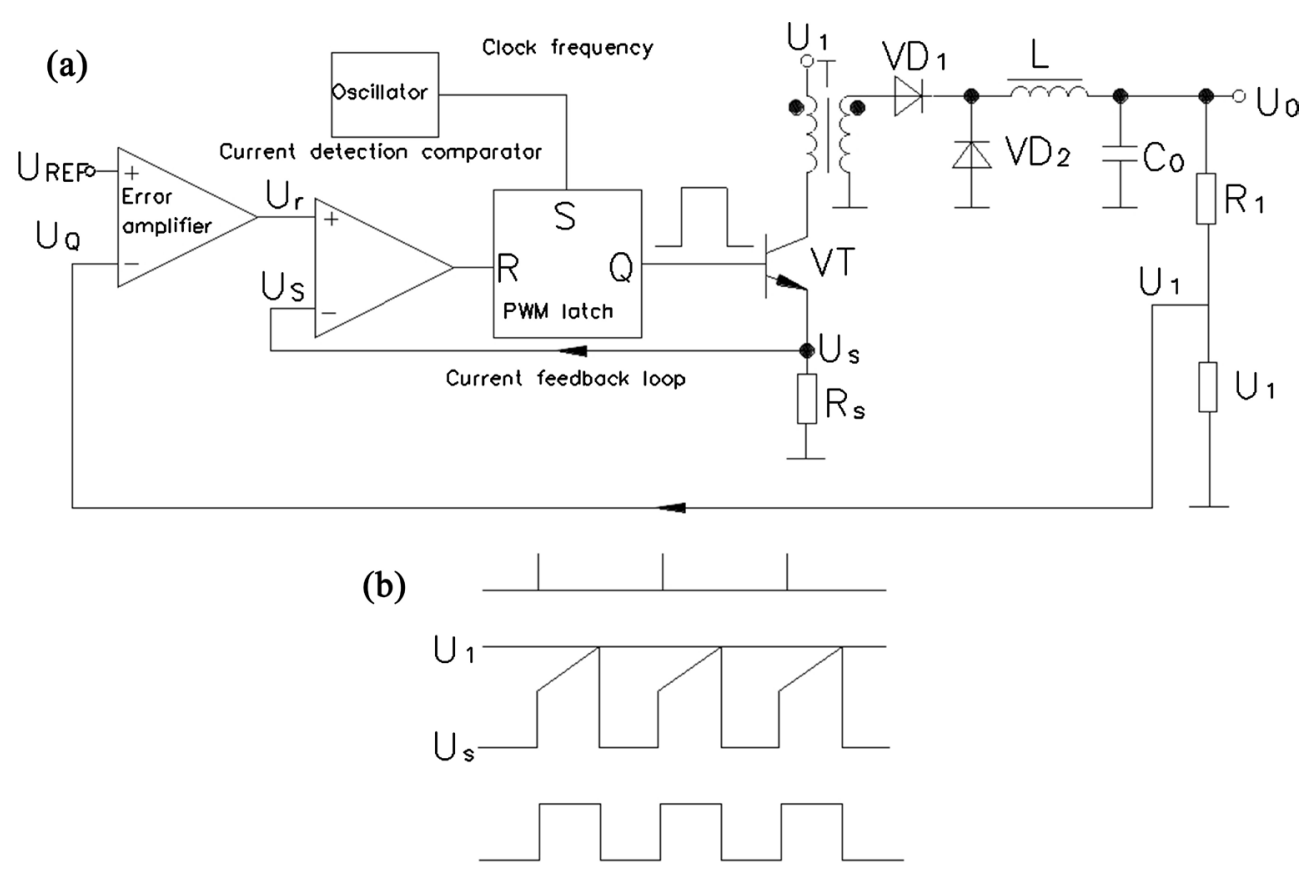

Figure 9. Waveform diagram of the output voltage $U$ and noise current of the pulse drive source. 


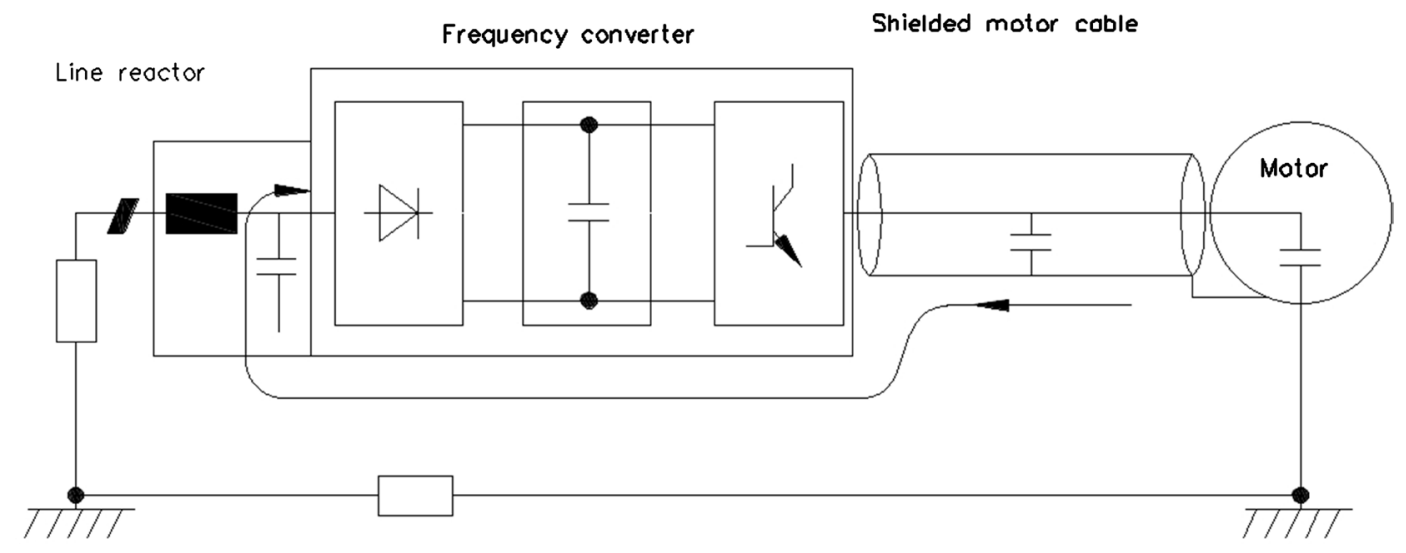

Figure 10. Noise current flow with shielded motor cable.

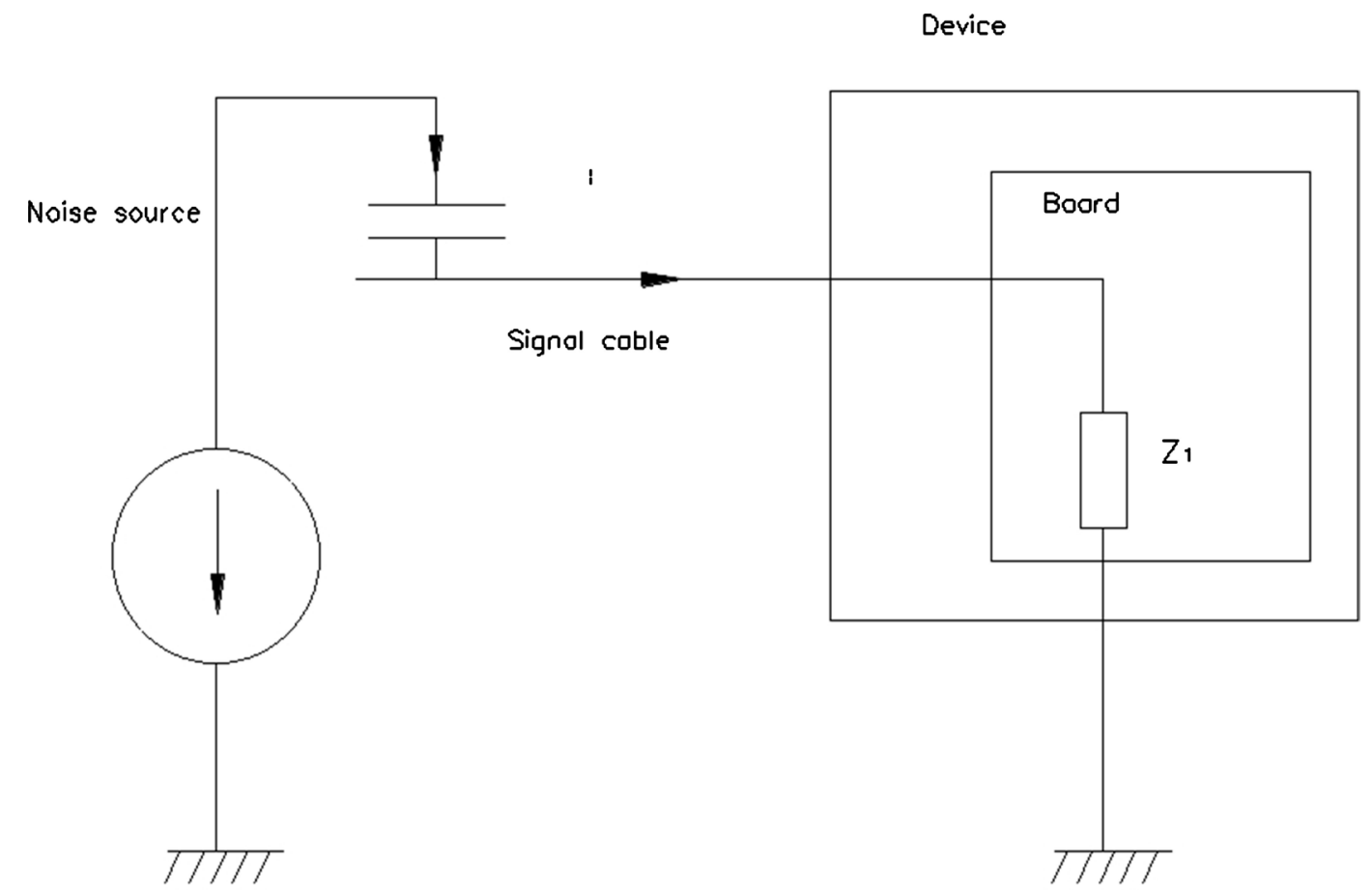

Figure 11. Coupling capacitance of unshielded signal cable.

pulse drive source becomes the noise receiver 1 . The most effective method is to strictly isolate the noise source and the signal line, and the shield at both ends of the signal line should be grounded, as shown in Figure 12.

\subsection{Electromagnetic Compatibility Design of Pulse Drive Source}

1) To ensure that all equipment is well grounded, it is necessary to make the ground wire short and thick, and then connect it to the ground. It is particularly important that all control devices (such as programmable automation equipment) connected to the pulse control source need to be in common ground and use short and thick wires when grounding. At the same time, the ground wire of the traction cable should be directly connected to the ground terminal (PE) of the coarse pulse control source. 


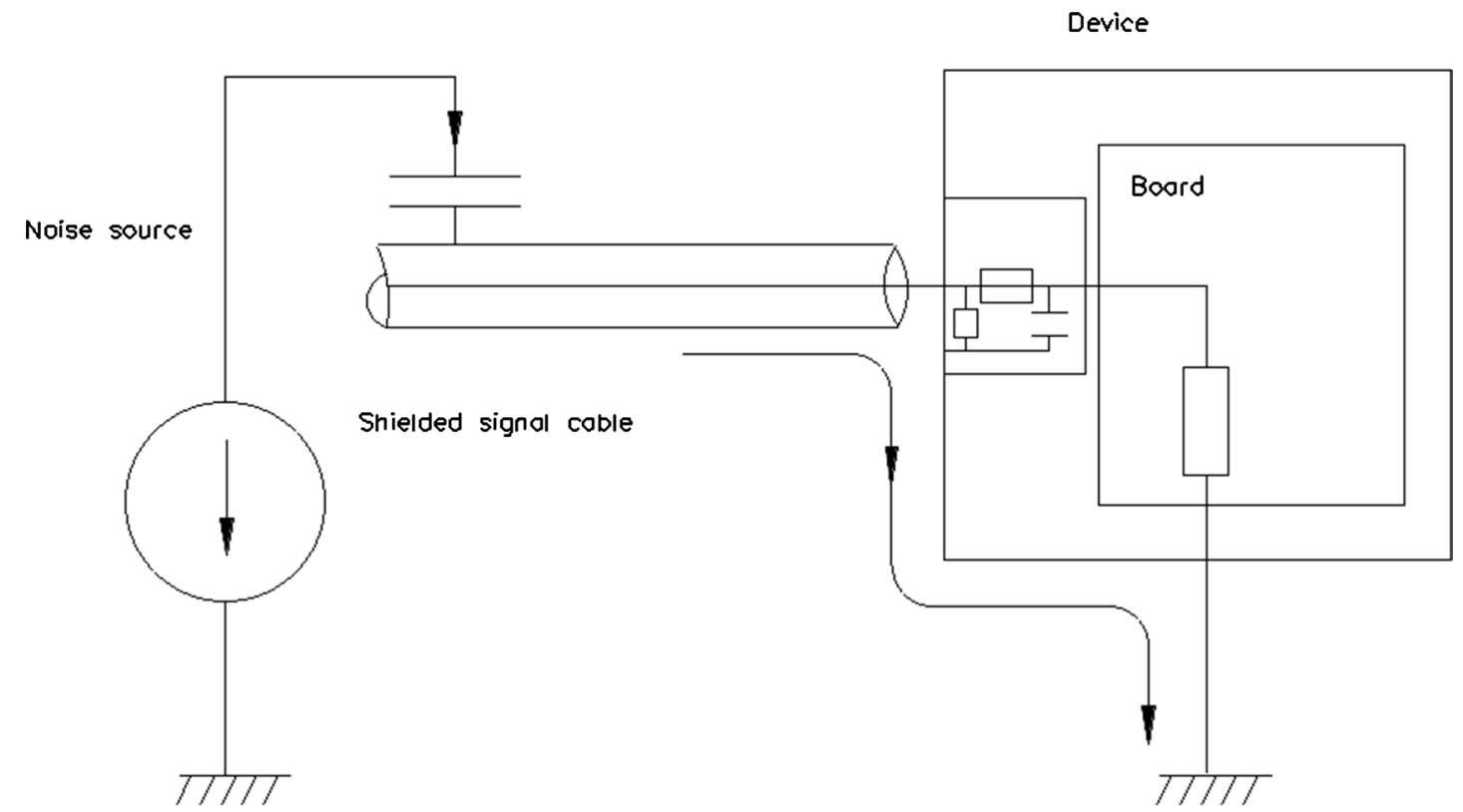

Figure 12. Use shielded signal cable to increase anti-interference.

2) In order to effectively suppress the radiation and conduction of electromagnetic waves, the cable driven by the pulse source should be a shielded cable, and the conductivity of the shielding layer should be at least one tenth of the conductivity of each phase conductor.

3) Shielded cables are best used for control cables. The analog signal transmission line should use double-shielded twisted wire. Different analog signal lines must operate independently and have their own shielding layer to reduce the coupling between the lines. Do not place different analog signals on the same common feedback line. It is best to use double-shielded twisted pair cables for low-voltage digital signal lines, or single-shielded twisted pair cables. Analog and digital signal transmission cables should be shielded and delivered separately.

4) The motor cable should be delivered separately from other cables. At the same time, the long distance parallel wiring of the motor cable and other cables should be avoided to reduce the electromagnetic interference caused by the rapid change of the output voltage of the pulse drive source.

5) If the pulse control source is used in a noise-sensitive environment, a filter can be used to reduce the radiated interference of the line.

\section{Conclusions}

Based on the existing work results, based on simulation and theoretical experience, this article uses the detailed theory and methods of electromagnetic compatibility, basic power electronics, modern electromagnetic fields and electromagnetic waves, combined with software and experimental methods to analyze thorough electromagnetic high power Pulse source drive, fundamentally clarified the electromagnetic compatibility mechanism of high-power pulse drive source. 
The manufacturing process of the high-power pulse control source is a combination of electromagnetic compatibility principle design and optimization. In this paper, high-frequency simulation software is used to simulate the high-frequency radiation of the high-power pulse drive source, and the radiation effect and shielding performance are analyzed. The results show that the electromagnetic compatibility of the control circuit part of the inverter is a problem that cannot be ignored. Although the problem of electromagnetic interference is smaller than that of the pulse control source part, because the pulse signal has strong electromagnetic interference, the part of the pulse signal needs to be strengthened. In this paper, the principle and composition of the pulse drive source system are elaborated, the main sources of electromagnetic interference of the pulse drive source are analyzed, and measures to improve electromagnetic compatibility are proposed. This paper studies and analyzes the electromagnetic compatibility of the high-power pulse control source from the perspective of theory and simulation.

\section{Conflicts of Interest}

The authors declare no conflicts of interest regarding the publication of this paper.

\section{References}

[1] Juntunen, R.D., Diedrich, D. and Krkoska, M. (2015) Power Transformation System.

[2] Yang, Y.H., Zhou, K. and Blaabjerg, F. (2016) Enhancing the Frequency Adaptability of Periodic Current Controllers with a Fixed Sampling Rate for Grid-Connected Power Converters. IEEE Transactions on Power Electronics, 31, 7273-7285.

[3] Eriksson, T., et al. (2011) Transformer Winding and a Method of Reinforcing a Transformer Winding.

[4] Wang, R.X., et al. (2010) Electrical Power System with High-Density Pulse Width Modulated (Pwm) Rectifier.

[5] Arashima, K. (2015) Sensor, Sensor Signal Processor, and Power Line Signal Encoder.

[6] Athab, H.S. and Lu, D.C. (2010) Simple Controller for Single-Phase Power Factor Correction Rectifier. IET Power Electronics, 3, 590-600.

https://doi.org/10.1049/iet-pel.2008.0320 